\title{
Sequencing the Regulations on Human Germline Editing Research
}

\author{
Nicholas Geringer ${ }^{1 *}$
}

Late last year, the world learned of one Chinese scientist named He Jiankui who covertly helped create the world's first gene-edited babies. While germline modifications in humans are currently prohibited across several countries worldwide, new committees such as the WHO Expert Advisory Committee on Developing Global Standards for Governance and Oversight of Human Genome Editing or the International Commission on the Clinical Use of Human Germline Genome Editing are being formed in hopes of developing frameworks that offer improved consensus regarding the current international inconsistencies surrounding human germline editing and mitigating the scientific community's point of concern for the risk of other researchers prematurely engaging in such research (Araki and Ishii, 2014). Although evaluating the consequences of improper or incorrect DNA modifications will always remain important in ensuring the safety of research participants in human gene editing research, the novelty of such work expresses an even greater concern for these ramifications. The guidelines in the Canadian Tri-Council Policy Statement provide a systematic framework for identifying ethical issues in research by evaluating three core principles: Respect for Persons, Concern for Welfare, and Justice (TCPS, 2014). This paper seeks to apply these principles in the context of the regulatory and legal frameworks regarding human germline editing and examine the critical nonscientific steps that must first be taken to ensure human subject research continues to operate in a safe and ethical manner even as advances in science and technology accelerate.

The principle of Respect for Persons is grounded in the idea of "recognizing the intrinsic value of human beings" (TCPS, 2014) by citing the moral obligations to respect autonomy and protect those with diminished autonomy. Consent is a critical component of autonomy, and more specifically, informed consent requires that foreseeable risks and potential benefits to both participants and others are adequately expressed (TCPS, 2014). Currently, several researchers and bioethicists recognize that while the risks of germline therapy remain largely unknown, informed consent cannot be obtained (Lanphier et al., 2015). Although all biomedical

\footnotetext{
${ }^{1}$ Faculty of Health Sciences, Queen's University, Kingston, Ontario, Canada, K7L 2V5
}

*To whom correspondence should be addressed: n.geringer@queensu.ca

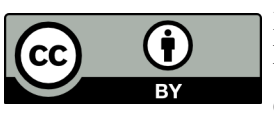

Except where otherwise noted, this work is licensed under https://creativecommons.org/licenses/by/4.0

doi:10.22186/jyi.37.2.22-23 research carries a degree of uncertainty and risk, human germline editing research specifically carries an incongruence between the individual(s) giving consent and the individual(s) that ultimately bear the consequences of the inherent risks: the child and, potentially, subsequent generations. It is understood that parents already make decisions that affect their future children, and research with children permits adults to assume the risks for the benefits of their offspring (National Human Genome Research Institute, 2017); however, the risks in these circumstances must be assessed "as no more than minimal" (Hebert, 2008). Drawing on language from the United States' general requirements for informed consent, "an explanation as to whether medical treatments are available if injury occurs" must be provided for any research that involves more than minimal risk (LII, 2005). However, due to the unknown nature of complications that may stem from off-target edits or mosaicism, an explanation for medical treatments to remedy such injuries cannot necessarily be attained (National Human Genome Research Institute, 2017). As such, additional non-human research examining potential inadvertent outcomes stemming from specific genes under consideration should be promoted prior to lifting moratoriums on human germline editing. Moreover, now that genetically modified babies exist, it is paramount that ethical longitudinal studies be launched on these children to inform future scientific and policy decisions and to ensure these children receive timely medical assistance should any complications arise. Gaining a greater understanding of the risks involved would better address concerns related to informed consent in human germline research and move closer towards meeting the TCPS' definition of Respect for Persons.

The purpose of promoting participant welfare is to minimize risk and maximize benefits for research participants, which is achieved primarily by ensuring that potential harms do not outweigh anticipated benefits, avoiding undue burdens, and clearly stating risks and benefits. Importantly, it is critical that the benefits of experimental research not be overstated in order to adequately protect participant welfare. It was discovered that the parents of the children whose genomes were edited by He were provided with "a consent form that falsely stated [his research] was an AIDS vaccine trial, and which conflated research with therapy by claiming they were "likely to benefit"' (Begley, 2018). Moving forward, it is imperative that research on human germline editing be explicitly defined as it is, rather than obscuring its purpose to motivate participants' consent under false pretenses. Human germline editing research differentiates itself from the remainder of human subject research due to the additional obligation to consider the implications both for the individual that lives with the manipulated genetic 
sequence and future generations that are prone to carrying these genetic alterations (NASEM, 2015). For this reason, transparency with regards to how the research is being represented is especially critical. Even more troublesome is the fact that such alterations would be nearly impossible to remove from the human population, especially ones caused by unintended modifications to ancillary target pathways (CIHR, 2016). Such considerations still fall under the purview of participant welfare, as the risks and potential benefits of research for the welfare of society must be equally evaluated alongside that of direct study participants (TCPS, 2014).

An examination of notions surrounding justice, which in the context of research refers to the duty to treat individuals fairly and equitably, is equally important. Beyond the scope of this piece, one of the strongest arguments against the use of human germline editing altogether remains the case of genetic enhancement, where gene editing may be used to select for certain traits or dispositions perceived as beneficial. While the focus of this paper is on research implications of germline editing in its current form, there are important considerations and numerous works that address whether the hypothetical application of such techniques should be restricted solely to correcting genetic anomalies. Returning to the issue of research, there is a drastic absence of liability that must be addressed prior to mainstream use of germline editing. Negligence was the charge laid in the unfortunate death of Jesse Gelsinger, the first person to die from unintended consequences of gene therapy, and the research institution was ultimately found to be liable with the case eventually being settled (Sibbald, 2001). However, the issue of liability remains contentious as the diffusion of responsibility in orchestrating such research across parties, including but not limited to researchers, the research institution, and government and private funding agencies, remains extremely challenging to always delineate (Ungar, 2011). As such, transparency in the reasoning and work of researchers and ethical research boards should be encouraged at every level, not only acting as a safeguard to increase the chances of detecting detrimental errors and intervening accordingly to minimize negative outcomes, but also ensuring that the assignment of liability in the event of a negative outcome is accurate and consistent by clarifying the roles and responsibilities of each of the parties outlined above. The TCPS (2014) cites an "imbalance of power ... between [the] researcher and participant" as an important threat to justice, as participants will have a weaker understanding of the research than the researcher. This supports the idea that, prior to engaging in human germline research, an appropriate level of oversight must be attained and the expectations of research parties made clear to ensure no one party is exercising undue influence or abusing power balances for the sake of expediting discovery.

Finally, central to what the future of germline editing may look like, the ethical underpinnings of such research must continuously be considered and re-evaluated. Some experts argue that once a threshold of safety and efficacy is established, using germline editing to eradicate diseases stemming from genetic abnormalities would be considered a moral imperative (Savulescu et al., 2015).
Nevertheless, other groups claim that even if ethically justifiable applications for this technology emerge, the argument in favor of its application remains moot until safe outcomes and reproducible data over several generations are demonstrated (Lanphier et al., 2015). While two genetically edited babies may have been successfully born, the long-term consequences, and success of the 'treatment' altogether, have yet to be demonstrated. Ultimately, it is imperative that the welfare and integrity of research participants take priority over all else in human research and, as the principle of Respect for Persons dictates, that individuals not be treated solely as means to achieving or furthering a research goal (TCPS, 2014). As research continues to expand in unprecedented ways, regulations, policies, and the law must be fluid to tackle novel concerns and adequately address the ethical, procedural, and legal principles of human research. While it can be reasonably inferred that we remain several years away from mainstream, legalized experimentation of germline editing in humans, it is crucial that efforts to better explore how human germline editing can be responsibly accomplished must be made on an ongoing basis consistent with emerging information to ensure the overall welfare of research participants remains appropriately and rightfully safeguarded.

\section{REFERENCES}

Araki, M. and Ishii, T. (2014). International regulatory landscape and integration of corrective genome editing into in vitro fertilization. Reproductive Biology and Endocrinology, 12(108), available: doi:10.1186/1477-7827-12-108.

Begley, S. (2018). 'Amid uproar, Chinese scientist defends creating gene-edited babies, 'STATNews, 28 Nov, available: https://www.statnews.com/2018/11/28/ chinese-scientist-defends-creating-gene-edited-babies/.

Canadian Institutes of Health Research. (2016). 'Human Germline Gene Editing: Points to Consider from a Canadian Perspective', available: http://www.cihrirsc.gc.ca/e/50158.html.

Canadian Institutes of Health Research, Natural Sciences and Engineering Research Council of Canada, and Social Sciences and Humanities Research Council of Canada. (2014). Tri-Council Policy Statement: Ethical Conduct for Research Involving Humans, available: http://www.pre.ethics.gc.ca/pdf/ eng/tcps2-2014/TCPS_2_FINAL_Web.pdf

Hebert, P. C. (2008). Doing Right. A Practical Guide to Ethics for Medical Trainees and Physicians, 3rd ed., Don Mills, Ontario: Oxford University Press Canada.

Lanphier, E., Urnov, F., Haecker, S. E., Werner, M., \& Smolenski, J. (2015). Don't edit the human germ line. Nature News, 519(7544), 410, available: doi:10.1038/519410a.

Legal Information Institute. (2005). 28 CFR 46.116 - General Requirements for Informed Consent, available: https://www.law.cornell.edu/cfr/text/28/46.116.

National Academies of Sciences Engineering Medicine. (2015). 'On Human Gene Editing: International Summit Statement', available: http://www8.nationalacademies.org/onpinews/newsitem.aspx?RecordID=12032015a.

National Human Genome Research Institute. (2017). What are the Ethical Concerns of Genome Editing?, available: https://www.genome.gov/27569225/ what-are-the-ethical-concerns-about-genome-editing/.

Savulescu, J., Pugh, J., Douglas, T., \& Gyngell, C. (2015). The moral imperative to continue gene editing research on human embryos. Protein Cell, 6, 476-479, available: doi:10.1007/s13238-015-0184-y.

Sibbald, B. (2001). Death but one unintended consequence of gene-therapy trial. CMAJ, 164(11), 1612

Ungar, D. (2011). The Canadian Bioethics Companion, available: http://canadi anbioethicscompanion.ca/the-canadian-bioethics-companion/chapter-8-research-ethics/\#top. 